TABLE 5-Continued

\begin{tabular}{|c|c|c|c|c|c|}
\hline$j$ & $F\left(x_{j}\right)=1 / 2+j / 256$ & $x_{j}$ & $j$ & $F\left(x_{j}\right)=1 / 2+j / 256$ & $x_{j}$ \\
\hline 65 & 0.75390625 & 0.68683375 & 97 & 0.87890625 & 1.16953661 \\
\hline 66 & 0.75781250 & 0.69928330 & 98 & 0.88281250 & 1.18916435 \\
\hline 67 & 0.76171875 & 0.71184220 & 99 & 0.88671875 & 1.20926123 \\
\hline 68 & 0.76562500 & $\begin{array}{lll}0.72451 & 438\end{array}$ & 100 & 0.89062500 & 1.22984876 \\
\hline 69 & 0.76953125 & 0.73730400 & 101 & $0.89453 \quad 125$ & $1.25099 \quad 172$ \\
\hline 70 & 0.77343750 & 0.75021538 & 102 & 0.89843750 & 1.27268865 \\
\hline 71 & 0.77734375 & 0.76325304 & 103 & 0.90234375 & 1.29502241 \\
\hline 72 & 0.78125000 & 0.77642176 & 104 & 0.90625000 & 1.31801090 \\
\hline 73 & $0.78515 \quad 625$ & 0.78972652 & 105 & 0.91015625 & 1.34171784 \\
\hline 74 & 0.78906250 & $0.80317 \quad 257$ & 106 & 0.91406250 & 1.36620382 \\
\hline 75 & 0.79296875 & 0.81676542 & 107 & 0.91796875 & 1.39153749 \\
\hline 76 & 0.79687500 & $\begin{array}{lll}0.83051 & 088\end{array}$ & 108 & 0.92187500 & 1.41779714 \\
\hline 77 & $0.80078 \quad 125$ & 0.84441508 & 109 & $0.92578 \quad 125$ & $1.44507 \quad 258$ \\
\hline 78 & 0.80468750 & $0.85848 \quad 447$ & 110 & 0.92968750 & 1.47345903 \\
\hline 79 & 0.80859375 & 0.87272589 & 111 & 0.93359375 & 1.50310294 \\
\hline 80 & 0.81250000 & 0.88714656 & 112 & 0.93750000 & 1.53412054 \\
\hline 81 & $0.81640 \quad 625$ & 0.90175411 & 113 & $0.94140 \quad 625$ & 1.56668859 \\
\hline 82 & 0.82031250 & 0.91655667 & 114 & 0.94531250 & 1.60100866 \\
\hline 83 & 0.82421875 & 0.93156283 & 115 & 0.94921875 & 1.63732538 \\
\hline 84 & 0.82812500 & 0.94678176 & 116 & 0.95312500 & 1.67594192 \\
\hline 85 & $0.83203 \quad 125$ & 0.96222320 & 117 & 0.95703125 & 1.71722812 \\
\hline 86 & 0.83593750 & 0.97789754 & 118 & 0.96093750 & 1.76167041 \\
\hline 87 & 0.83984375 & 0.99381591 & 119 & 0.96484375 & 1.80989233 \\
\hline 88 & 0.84375000 & 1.00999017 & 120 & 0.96875000 & $\begin{array}{lll}1.86273 & 187\end{array}$ \\
\hline 89 & $0.84765 \quad 625$ & 1.02643306 & 121 & 0.97265625 & 1.92135077 \\
\hline 90 & 0.85156250 & 1.04315826 & 122 & 0.97656250 & 1.98742789 \\
\hline 91 & 0.85546875 & 1.06018048 & 123 & 0.98046875 & 2.06352790 \\
\hline 92 & 0.85937500 & 1.07750557 & 124 & 0.98437500 & 2.15387469 \\
\hline 93 & $0.86328 \quad 125$ & 1.09518065 & 12 & $0.98828 \quad 125$ & 2.26622681 \\
\hline 94 & 0.86718750 & 1.11319428 & 126 & 0.99218750 & 2.41755902 \\
\hline 95 & 0.87109375 & 1.13157656 & 127 & 0.99609375 & 2.66006747 \\
\hline 96 & 0.87500000 & 1.1503593 & & & \\
\hline
\end{tabular}

\title{
Algebraic Approximations for Laplace's Equation in the Neighborhood of Interfaces
}

\author{
By J. W. Sheldon
}

1. Introduction. Let it be required to solve the following problem:

Problem A:

Let $C_{i}, i=1,2$, be two simple, closed plane curves with continuous curvature. Let $C_{2}$ enclose all the points of $C_{1}$. Let $G_{1}$ be the region interior to $C_{1}$. Let $G_{2}$ be the region interior to $C_{2}$ and exterior to $C_{1}$. Let $W$ be a continuous, bounded function of position on $C_{2}$. Let it be required to find harmonic functions $V^{(i)}$ such that

(a) $V^{(2)}=W$ on $C_{2}$.

(b) $V^{(i)}$ is regular and bounded in $G_{i}$.

Received September 19, 1957. 
(c) $V^{(1)}=V^{(2)}$ on $C_{1}$.

(d) $D_{1} \frac{\partial V^{(1)}}{\partial n}=D_{2} \frac{\partial V^{(2)}}{\partial n}$ on $C_{1}$, where $D_{i}>0$ and $\frac{\partial}{\partial n}$ denotes differentiation along the normal to $C_{1}$, the positive normal direction being chosen as the direction from $G_{1}$ into $G_{2}$.

Problems with boundary conditions (c) and (d) connecting distinct harmonic functions on a common boundary between adjacent regions arise in electrostatics, and specific cases are treated in books dealing with electrostatic theory. (See, for example, Jeans [1] and Smythe [2].) For electrostatics, $D_{1}$ and $D_{2}$ are the dielectric constants in $G_{1}$ and $G_{2}$. Boundary condition (c) in electrostatic theory is derived from the requirement that no work be done taking a charge around a closed path which crosses $C_{1}$. The required condition is found to be $V^{(1)}=V^{(2)}$ +"constant" and we set "constant" to zero. Boundary condition (d) is derived from Gauss's electric flux theorem. Boundary condition (d) may also be derived by a variational method. If we require that $D_{1}$ times the Dirichlet integral for $G_{1}$ plus $D_{2}$ times the Dirichlet integral for $G_{2}$ be an extremal, and that $V^{(1)}=V^{(2)}$ on $C_{1}$, then (d) is obtained as the natural boundary condition on $C_{1}$.

Problem A may also arise for steady-state heat conduction, steady-state diffusion, steady-state current flow, and for problems of incompressible, immiscible fluid flow in porous media. In the latter problem $C_{1}$ may be a moving curve [3]. Boundary conditions (c) and (d) may also arise in transient problems of diffusion type.

We suppose that we wish to solve Problem A by finite difference methods. We introduce a rectangular network over a region covering $G_{1}$ and $G_{2}$. We introduce a function $\varphi$, defined at each meshpoint interior to $C_{2}$, which is to approximate in some way the solution of Problem A. In order to determine $\varphi$ we require a system of equations equal in number to the number of meshpoints interior to $C_{2}$. We call each equation in the system an "algebraic approximation." In the way that we will treat this problem, there will be an algebraic approximation associated with each meshpoint inside $C_{2}$ in the sense that each algebraic approximation approximates to the conditions of Problem A best in the neighborhood of a unique meshpoint and no two algebraic approximations approximate Problem A best in the neighborhood of the same meshpoint. The meshpoints interior to $C_{2}$ may be classified in the following way:

(i) Meshpoints adjacent to $C_{2}$. A meshpoint is adjacent to $C_{2}$ if a straight-line segment from the meshpoint to one of the four nearest neighbor meshpoints intersects or extends to $C_{2}$.

(ii) Meshpoints adjacent to $C_{1}$. A meshpoint is adjacent to $C_{1}$ if the meshpoint is situated on $C_{1}$ or if a straight-line segment from the meshpoint to one of the four nearest neighbor meshpoints intersects $C_{1}$ (but not if the segment only extends to $C_{1}$ ).

(iii) Normal meshpoints. All other meshpoints interior to $C_{2}$ are called normal meshpoints.

The algebraic approximation to be associated with the normal meshpoints is the well-known five-point formula for approximating the Laplace equation. 
Algebraic approximations at the meshpoints adjacent to $C_{2}$ have been obtained. See, for example, Shortley and Weller [4] or Viswanathan [5] (Algebraic approximations have been obtained for Dirichlet or Neuman or mixed conditions on $C_{2}$.) The present paper is concerned with obtaining algebraic approximations for meshpoints adjacent to $C_{1}$.

Problems with boundary conditions (c) and (d) of Problem A have been treated by finite difference methods in connection with the "group-diffusion" problems of reactor design $[6,7]$. To the author's knowledge, in this field it has always been assumed that the interfaces between materials have contours such that analogues of equations (21) or (22) apply. If the interface does not meet the conditions for equations (21) or (22), it has been customary to perturb the interface so that it does. In some problem this may be an unsatisfactory procedure. In the case of "moving boundary" problems, for example, the essence of the problem consists in getting the velocity of the moving boundary accurately.

2. Existence of solution to Problem A. Books on potential theory which give existence proofs for the Neuman and Dirichlet problems do not (to the author's knowledge) treat Problem A. An existence proof can be constructed following the integral equation technique described by Kellogg [8]. This method consists in postulating charge and dipole distributions on the boundary curves, and then obtaining and proving the existence of a solution of the equations which must be satisfied if the charge and dipole distributions are to produce potential functions which satisfy the given boundary conditions. The equations obtained are linear integral equations. The method can be extended to show that Problem A possesses a solution. It can be shown that $V^{(2)}$ is the potential of a continuous dipole distribution on $C_{2}$ plus the potential of a continuous charge distribution on $C_{1}$, $V^{(1)}$ is the potential of a continuous charge distribution on $C_{1}$. (The charge distribution on $C_{1}$ to produce $V^{(1)}$ is different from that on $C_{1}$ which contributes to produce $V^{(2)}$.) We shall not give the detailed proof. In view of the fact that Problem $\mathrm{A}$ is the solution of a problem in the calculus of variations, and that we have required $C_{1}$ and $C_{2}$ to be smooth and $W$ to be continuous, it would be very surprising if the solution did not exist. Hence we would not really be justified in presenting a rather long existence proof here.

3. The behavior of $V^{(1)}$ and $V^{(2)}$ on $C_{1}$. Let us suppose that $C_{1}$ is an analytic arc or that $C_{1}$ is composed of segments of analytic arcs. We have the following:

TheOREM 1. A segment $\Gamma$ of $C_{1}$ which is an analytic arc is not a natural boundary for $V^{(1)}$ or $V^{(2)}$.

This means that every point of $\Gamma$ has a neighborhood such that there exists a harmonic function which is regular in the region consisting of $G_{1}$ and this neighborhood which coincides in $G_{1}$ with $V^{(1)}$. And every point of $\Gamma$ has a neighborhood such that there exists a harmonic function which is regular in the region $G_{2}$ and this neighborhood and which coincides in $G_{2}$ with $V^{(2)}$.

In order to prove Theorem 1 we first solve the following problem:

Problem B. Let $K$ be a circle of radius $R$ and center at the origin of the $x, y$ coordinate system. Let $\bar{G}_{1}$ be the region interior to $K$ lying below the $x$-axis and let $\bar{G}_{2}$ be the region interior to $K$ lying above the $x$-axis. Call the part of the $x$-axis 
interior to $K, \bar{\Gamma}$. Let $r, \theta$ be polar coordinates in the $x, y$ plane. Let $W^{(1)}(\theta)$, $-\pi \leq \theta \leq 0, W^{(2)}(\theta), 0 \leq \theta \leq \pi$ be given bounded continuous functions of $\theta$ on $K$ satisfying $W^{(1)}(0)=W^{(2)}(0), W^{(1)}(-\pi)=W^{(2)}(\pi), D_{1} \frac{\partial W^{(1)}(0)}{\partial \theta}=D_{2} \frac{\partial W^{(2)}(0)}{\partial \theta}$, $D_{1} \frac{\partial W^{(1)}(-\pi)}{\partial \theta}=D_{2} \frac{\partial W^{(2)}(\pi)}{\partial \theta}$. Let it be required to find functions $\bar{V}^{(i)}$ such that

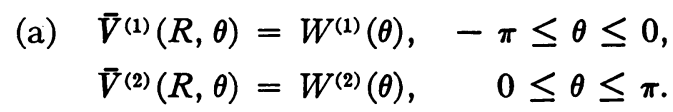

(b) $\bar{V}^{(i)}$ is regular and bounded in $\bar{G}^{(i)}$.

(c) $\quad \bar{V}^{(1)}(r, \theta)=\bar{V}^{(2)}(r, \theta)$ on $\bar{\Gamma}$.

(d) $D_{1} \frac{\partial \bar{V}^{(1)}(r, \theta)}{\partial \theta}=D_{2} \frac{\partial \bar{V}^{(2)}(r, \theta)}{\partial \theta}$ on $\bar{\Gamma}$.

Problem B can be solved by the method of circular harmonics. We obtain :

(1) $\bar{V}^{(1)}(r, \theta)=\frac{1}{2} A_{0}+\sum_{n=1}^{\infty} A_{n}\left(\frac{r}{R}\right)^{n} \cos n \theta$ $+\sum_{n=1}^{\infty} B_{n}\left(\frac{r}{R}\right)^{n} \sin n \theta, \quad-\pi \leq \theta \leq 0$,

(2) $\bar{V}^{(2)}(r, \theta)=\frac{1}{2} A_{0}+\sum_{n=1}^{\infty} A_{n}\left(\frac{r}{R}\right)^{n} \cos n \theta$

$$
+\frac{D_{1}}{D_{2}} \sum_{n=1}^{\infty} B_{n}\left(\frac{r}{R}\right)^{n} \sin n \theta, 0 \leq \theta \leq \pi,
$$

$$
A_{m}=\frac{2 D_{1}}{\left(D_{1}+D_{2}\right) \pi} \int_{-\pi}^{0} W^{(1)}(\theta) \cos m \theta d \theta
$$

$$
+\frac{2 D_{2}}{\left(D_{1}+D_{2}\right) \pi} \int_{0}^{\pi} W^{(2)}(\theta) \cos m \theta d \theta
$$

$$
B_{m}=\frac{2 D_{2}}{\left(D_{1}+D_{2}\right) \pi}\left[\int_{-\pi}^{0} W^{(1)}(\theta) \sin m \theta d \theta+\int_{0}^{\pi} W^{(2)}(\theta) \sin m \theta d \theta\right] \text {, }
$$

$$
m=0,1,2 \cdots \text {. }
$$

With (3), (4) substituted into (1) and $r=R$, it is apparent that (1) represents the sum of two Fourier cosine series plus two Fourier sine series. The formal sums of the two series are easily identified. The two cosine series give us

$$
\frac{D_{1}}{D_{1}+D_{2}} W^{1}(\theta)+\frac{D_{2}}{D_{1}+D_{2}} W^{2}(-\theta), \quad-\pi \leq \theta \leq 0 .
$$

The two sine series give us

$$
\frac{D_{2}}{D_{1}+D_{2}} W^{1}(\theta)-\frac{D_{2}}{D_{1}+D_{2}} W^{2}(-\theta), \quad-\pi \leq \theta \leq 0 .
$$

Adding the above terms, the sum of the two cosine series plus the two sine series gives us $W^{1}(\theta),-\pi \leq \theta \leq 0 . W^{1}(\theta), W^{2}(\theta)$ satisfy Dirichlet conditions and are 
furthermore continuous within their range of definition. This means that the cosine series converge to their formal sums for $-\pi \leq \theta \leq 0$, and the sine series converge to their formal sums for $-\pi<\theta<0$. The sine series converge to the value zero, at $\theta=0, \theta=\pi$. The sum of the four series gives $W^{1}(\theta)$ for $-\pi \leq \theta \leq 0$. A similar argument shows that if $r=R$ the series on the right-hand side of (2) converges to $W^{2}(\theta)$ for $0 \leq \theta \leq \pi$. It is readily verified that $\bar{V}^{(1)}(r, \theta), \bar{V}^{(2)}(r, \theta)$ as defined by the series in (1), (2) satisfy the remaining conditions of Problem $B$ so that (1), (2) is the solution of Problem B. Then we have:

LeMma 1. $\bar{\Gamma}$ is not a natural boundary for $\bar{V}^{(1)}(r, \theta)$ or $\bar{V}^{(2)}(r, \theta)$ of Problem $B$.

The series in (1) and (2) are each uniformly convergent for $r \leq \rho \leq R$ for all $\theta$ and hence each of (1), (2) defines a harmonic function throughout the interior of $K$. Q.E.D.

It is, of course, a well known theorem from function theory that under a conformal transformation a harmonic function maps into a harmonic function. We have:

LEMMA 2. Let a neighborhood of $C_{1}$ be mapped into a neighborhood of $\bar{C}_{1}$, where $\bar{C}_{1}$ is the mapping of $\bar{C}_{1}$, by a conformal transformation. Let $V^{(i)}$ map into $\bar{V}^{(i)}$. Then $D_{1} \frac{\partial \bar{V}^{(1)}}{\partial n}=D_{2} \frac{\partial \bar{V}^{(2)}}{\partial n}$ on $\bar{C}_{1}$.

The Lemma follows from the definition of a conformal transformation.

With the help of the solution to Problem B and Lemmas 1, 2 we can prove Theorem 1. The method of proof follows closely a proof by Bieberbach [9] of a lemma from potential theory to the effect that if $V$ were regular in a region $G$, continuous in the extended domain obtained by adding to $G$ the points of an analytic arc that belongs to the boundary of $G$ and if $V$ were zero on this arc, then this arc is not a natural boundary for $V$. We may consider $V^{(i)}$ to be the real or imaginary part of a function of a complex variable. Let the analytic arc $\Gamma$ be represented in the complex plane by $z(\alpha), a \leq \alpha \leq b$, where $\alpha$ is the real part of a complex variable $\gamma=\alpha+i \beta . \alpha$ is also chosen as the arc length along $\Gamma$.

Then the function $z(\gamma)$ maps a neighborhood $K$ in the $\gamma$-plane of every point $\beta=0, a \leq \alpha_{0} \leq b$ onto a neighborhood $U$ of the point $z=z\left(\alpha_{0}\right)$; this mapping is conformal. Since $\alpha$ represents arc length along $\Gamma x^{\prime}\left(\alpha_{0}\right)^{2}+y^{\prime}\left(\alpha_{0}\right)^{2}=1$. Hence $z^{\prime}\left(\alpha_{0}\right) \neq 0$. Hence the mapping is also simple. We choose for $K$ a circle with center on the real axis at $\alpha_{0}$. This determines a region $U$ in the $z$-plane. Let $G_{i}$ and $V^{(i)}$, $i=1,2$, refer to the regions and functions of Problem A. From the intersection of $G_{i}$ and the boundary of $U$ we obtain the values of $V^{(i)}$ on the boundary of $U$. By the inverse of the mapping mentioned the boundary values on $U$ are transformed to boundary values on $K$. These satisfy the conditions for Problem B. From the results of Problem B each of the transforms of the functions $V^{(i)}$ to $K$ is analytic in $K$, and hence by an inverse transformation each of the $V^{(i)}$ is analytic throughout $U$. Q.E.D.

The domain of analyticity of the functions continued across $\Gamma$ is difficult to determine. It will depend on the location of the zeros and poles of $z(\gamma)$, as well as on the location of non-analytic points of $C_{1}$, and the distance of $C_{1}$ to $C_{2}$, and also the location of the mapping of $C_{2}$.

4. The connection between power series for $\bar{V}^{(1)}$ and $\bar{V}^{(2)}$. Let $O$ be the center of the circle $K$. Let $t$ and $n$ be the abscissa and ordinate measured from $O$. We 
label the value of $\bar{V}^{(i)}$ at $O, \bar{V}_{0}^{(i)}$ and we let $\bar{V}_{t}^{i}, \bar{V}_{n}^{(i)}, \bar{V}_{t i}^{(i)}$, etc., be partial derivatives of $\bar{V}^{(i)}$ with respect to $t$ and $n$ at $O$. We may expand $\bar{V}^{(i)}$ about $O$ in power series convergent in $K$ :

$$
\begin{aligned}
& \bar{V}^{(1)}=\bar{V}_{0}^{(1)}+\bar{V}_{n}^{(1)} n+\bar{V}_{l}^{(1)} t+\frac{1}{2} \bar{V}_{n n}^{(1)} n^{2}+\bar{V}_{n t}^{(1)} n t+\frac{1}{2} \bar{V}_{l t}^{(1)} t^{2}+\cdots, \\
& \bar{V}^{(2)}=\bar{V}_{0}^{(2)}+\bar{V}_{n}^{(2)} n+\bar{V}_{t}^{(2)} t+\frac{1}{2} \bar{V}_{n n}^{(2)} n^{2}+\bar{V}_{n t}^{(2)} n t+\frac{1}{2} \bar{V}_{t l}^{(2)} t^{2}+\cdots,
\end{aligned}
$$

THEOREM 2. If the power series for $\bar{V}^{(1)}$ is given the coefficients in the power series for $\bar{V}^{(2)}$ may be determined.

Since $\bar{V}^{(2)}=\bar{V}^{(1)}$ on $\Gamma$ it follows that

$$
\bar{V}_{t}^{(2)}=\bar{V}_{t}^{(1)}, \quad \bar{V}_{t t}^{(2)}=\bar{V}_{t t}^{(1)}
$$

Since $D_{2} \bar{V}_{n}^{(2)}=D_{1} \bar{V}_{n}^{(1)}$, it follows that

$$
D_{2} \bar{V}_{n t}^{(2)}=D_{1} \bar{V}_{n t}^{(1)}
$$

Since $\bar{V}_{t i}^{(2)}=\bar{V}_{t i}^{(1)}, \bar{V}_{t i}^{(i)}+\bar{V}_{n n}^{(i)}=0, i=1,2$, it follows that $\bar{V}_{n n}^{(2)}=\bar{V}_{n n}^{(1)}$. We have expressed $\bar{V}^{(2)}$ and all its derivatives through second order in terms of $\bar{V}^{(1)}$ and its derivatives through second order. Let $\xi=D_{1} / D_{2}$. We have

$$
\bar{V}^{(2)}=\bar{V}_{0}^{(1)}+\xi \bar{V}_{n}^{(1)} n+\bar{V}_{t}^{(1)} t+\frac{1}{2} \bar{V}_{n n}^{(1)} n^{2}+\xi \bar{V}_{n t}^{(1)} n t+\frac{1}{2} \bar{V}_{t t} t^{2} .
$$

We shall be concerned with terms through second order only. However, one may show that (7), (8) and the derivatives of the equations $V_{t i}^{(i)}+V_{n n}^{(i)}=0$ always give enough equations so that the derivatives of $\bar{V}^{(2)}$ can be expressed in terms of those of $\bar{V}^{(1)}$.

5. Five point algebraic approximations. We consider first the case in which $\Gamma$ is, locally, a straight line. We suppose that $\Gamma$ passes through a meshpoint making an angle $\alpha$ with the $x$-axis. Let the meshpoint be labeled " $a$ " and let the four nearest neighbors be labeled " $b$, , " $c$," " $d$, , " $e$ " as shown in figure 1. From Theorem 1 it follows that $V^{(i)}$ should satisfy Laplace's equation at " $a$." Let the

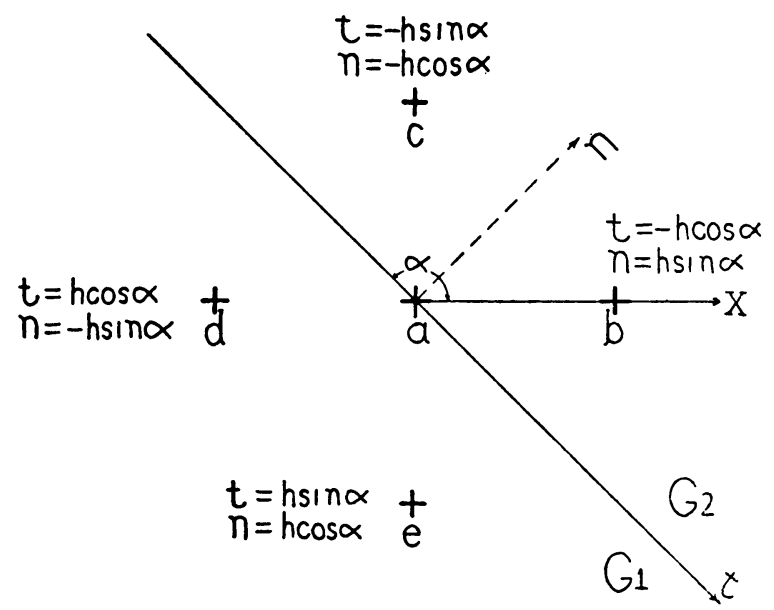

FIgURE 1. 
network values of $V^{(i)}$ at $a, b, c, d, e$ be $V_{a}, V_{b}, V_{c}, V_{d}, V_{e}$. It is not necessary to superscript these quantities since there is only one function value associated with each meshpoint. Let $h$ be the mesh spacing. We have the following theorem:

THEOREM 3. When $\xi \neq 1$, except for $\alpha=\frac{n \pi}{4}, n=0,1, \cdots, 7$, there exists no algebraic approximation for Laplace's equation in terms of $V_{a}, V_{b}, V_{c}, V_{d}, V_{e}$ which is such that the local truncation error tends to zero as $h \rightarrow 0$.

For definiteness, assume that $\pi / 2 \leq \alpha \leq \pi$. Then meshpoints $b$ and $c$ are on the boundary of or inside $G_{2}$ and $d$ and $e$ are on the boundary of or inside $G_{1}$.

We choose an origin at " $a$ " and introduce coordinates $t, n$ where $n$ is the normal at " $a$ " from $G_{1}$ into $G_{2}$ and $t$ is the distance along $\Gamma$ from $a$. The direction of positive $t$ is chosen so that the $t, n$ coordinate system is right-handed. It is assumed that a circle about $a$ which just encloses $b, c, d, e$ has radius less than the circle of convergence of the power series (5), (6) and that $\Gamma$ is straight inside this circle. Since $\Gamma$ is straight it is not necessary to distinguish between $\Gamma, \bar{\Gamma} ; V^{(i)}$, $\bar{V}^{(i)}$, etc. The values assumed by $t$ and $n$ at $b, c, d, e$ are given in figure 1. Partial derivatives $V_{n}, V_{t}$, etc., refer to $V_{n}^{(1)}, V_{t}^{(1)}$, etc., in what follows. Making use of (5), (9) we have

$$
\begin{aligned}
& V_{b}-V_{a}=\xi h \sin \alpha V_{n}-h \cos \alpha V_{t}+\frac{1}{2} h^{2} \sin ^{2} \alpha V_{n n} \\
& -\xi h^{2} \sin \alpha \cos \alpha V_{n t}+\frac{1}{2} h^{2} \cos ^{2} \alpha V_{t t}+O\left(h^{8}\right), \\
& V_{c}-V_{a}=-\xi h \cos \alpha V_{n}-h \sin \alpha V_{t}+\frac{1}{2} h^{2} \cos ^{2} \alpha V_{n n} \\
& +\xi h^{2} \sin \alpha \cos \alpha V_{n t}+\frac{1}{2} h^{2} \sin ^{2} \alpha V_{t t}+O\left(h^{3}\right), \\
& V_{d}-V_{a}=-h \sin \alpha V_{n}+h \cos \alpha V_{t}+\frac{1}{2} h^{2} \sin ^{2} \alpha V_{n n} \\
& -h^{2} \sin \alpha \cos \alpha V_{n t}+\frac{1}{2} h^{2} \cos ^{2} \alpha V_{t t}+O\left(h^{3}\right), \\
& V_{e}-V_{a}=h \cos \alpha V_{n}+h \sin \alpha V_{t}+\frac{1}{2} h^{2} \cos ^{2} \alpha V_{n n} \\
& +h^{2} \sin \alpha \cos \alpha V_{n t}+\frac{1}{2} h^{2} \sin ^{2} \alpha V_{t t}+O\left(h^{3}\right), \\
& O=V_{n n}+V_{t t}
\end{aligned}
$$

Suppose we could solve (10)-(13) for $V_{n n}$ and $V_{t t}$. By substituting the solution into (14) we would have an algebraic approximation to Laplace's equation. The $O\left(h^{3}\right)$ terms would be $O(h)$ after carrying out this process and would tend to zero as $h \rightarrow 0$. A necessary condition for being able to carry out this process is that the determinant of coefficients of (10)-(14) vanish. In order to evaluate the determinant, we take the following combinations of equations (10)-(14) :

$$
\begin{aligned}
& \text { Eq. } 15=\text { Eqs. }\left[(10)+(11)+(12)+(13)-h^{2}(14)\right], \\
& \text { Eq. } 16=\text { Eqs. }\left[(10)+(11)+\xi(12)+\xi(13)-\frac{1+\xi}{2} h^{2}(14)\right], \\
& \text { Eq. } 17=\text { Eqs. }\left[(11)-\frac{1}{2} \sin ^{2} \alpha h^{2}(14)+\xi(12)-\frac{\xi}{2} \cos ^{2} \alpha h^{2}(14)\right], \\
& \text { Eq. } 18=\text { Eqs. }\left[(11)+(12)-\frac{1}{2} h^{2}(14)\right], \\
& \text { Eq. } 19=\text { Eq. }(14) .
\end{aligned}
$$


The transformation of (11)-(14) to (15)-(19) is non-singular for $\xi \neq 1$. We obtain

$$
\begin{aligned}
& (\xi-1)(\sin \alpha-\cos \alpha) h V_{n}=V_{b}+V_{c}+V_{d}+V_{e}-4 V_{a}+O\left(h^{3}\right), \\
& (\xi-1)(\sin \alpha+\cos \alpha) h V_{t}=V_{b}+V_{c}-2 V_{a} \\
& +\xi\left(V_{d}+V_{e}-2 V_{a}\right)+O\left(h^{3}\right), \\
& \text { (17) }-\xi(\sin \alpha+\cos \alpha) h V_{n}+(\xi \cos \alpha-\sin \alpha) h V_{t} \\
& +\frac{1-\xi}{2}\left(\cos ^{2} \alpha-\sin ^{2} \alpha\right) h^{2} V_{n n}=V_{c}-V_{a}+\xi\left(V_{d}-V_{a}\right)+O\left(h^{3}\right) \text {, } \\
& \text { (18) }-(\sin \alpha+\xi \cos \alpha) h V_{n}+(\cos \alpha-\sin \alpha) h V_{t} \\
& +(\xi-1) \sin \alpha \cos \alpha h^{2} V_{n t}=V_{c}+V_{d}-2 V_{a}+O\left(h^{3}\right), \\
& \text { (19) } V_{n n}+V_{t t}=0 \text {. }
\end{aligned}
$$

The equation system (15)-(19) is triangular. Its determinant $D$ is

$$
D=\frac{1}{2}(\xi-1)^{4}\left(\sin ^{2} \alpha-\cos ^{2} \alpha\right)^{2} \sin \alpha \cos \alpha h^{6} .
$$

$D$ is zero for $\xi \neq 1, \frac{\pi}{2} \leq \alpha \leq \pi$ only when $\alpha=\frac{\pi}{2}, \alpha=\frac{3 \pi}{4}, \alpha=\pi$. By symmetry, $D$ would be zero also only for $\alpha=\frac{n \pi}{4}, n=0,1, \cdots, 7$ for $0 \leq \alpha<2 \pi$.

This proves Theorem 3. The algebraic approximations which we get, for $\alpha=\frac{3 \pi}{4}$ and $\alpha=\pi$ are, respectively:

$$
\begin{aligned}
V_{t i}^{(i)}+V_{n n}^{(i)}= & \frac{2 D_{2}}{D_{1}+D_{2}}\left[\frac{V_{b}-2 V_{a}+V_{c}}{h^{2}}\right] \\
& +\frac{2 D_{1}}{D_{1}+D_{2}}\left[\frac{V_{d}-2 V_{a}+V_{e}}{h^{2}}\right]+O(h)=0 \\
V_{t i}^{(i)}+V_{n n}^{(i)}= & \frac{V_{b}-2 V_{a}+V_{d}}{h^{2}}+\frac{2 D_{2}}{D_{1}+D_{2}}\left(\frac{V_{c}-V_{a}}{h^{2}}\right) \\
& \quad+\frac{2 D_{1}}{D_{1}+D_{2}}\left(\frac{V_{e}-V_{a}}{h^{2}}\right)+O(h)=0
\end{aligned}
$$

(21) and (22) may also be obtained by an application of Gauss's theorem $[6,7]$.

If $\Gamma$ is straight but does not pass through meshpoints, we still obtain an algebraic approximation in terms of four neighbors if $\alpha=\frac{n \pi}{2}$. For example, suppose $\alpha=\pi$ and $\Gamma$ passes a distance $\eta$ above " $a$ " leaving " $a$ " in $G_{1}$, " $c$ " in $G_{2}$. We choose an origin $O$ on $\Gamma$ where $\Gamma$ intersects $a c$. We let $V_{t}, V_{n}$, etc., represent derivatives of $V^{(1)}$ at $O$. The continuation equations analogous to (10)-(13) are

$$
\begin{aligned}
& V_{a}-V_{0}=-\eta V_{n}+\frac{1}{2} \eta^{2} V_{n n}+O\left(h^{3}\right), \\
& V_{b}-V_{0}=-\eta V_{n}+h V_{t}+\frac{1}{2} \eta^{2} V_{n n}-h \eta V_{n t}+\frac{1}{2} h^{2} V_{t t}+O\left(h^{3}\right),
\end{aligned}
$$




$$
\begin{aligned}
& V_{c}-V_{0}=\xi(h-\eta) V_{n}+\frac{1}{2}(h-\eta)^{2} V_{n n}+O\left(h^{3}\right), \\
& V_{d}-V_{0}=-\eta V_{n}-h V_{t}+\frac{1}{2} \eta^{2} V_{n n}+h \eta V_{n t}+\frac{1}{2} h^{2} V_{t t}+O\left(h^{3}\right), \\
& V_{e}-V_{0}=-(\eta+h) V_{n}+\frac{1}{2}(h+\eta)^{2} V_{n n}+O\left(h^{3}\right) .
\end{aligned}
$$

Subtracting (23) from (25) we obtain

$$
V_{c}-V_{a}=[\xi(h-\eta)+\eta] V_{n}+\frac{1}{2}\left(h^{2}-2 h \eta\right) V_{n n}+O\left(h^{3}\right) .
$$

Subtracting (27) from (23)

$$
V_{a}-V_{e}=h V_{n}-\frac{1}{2}\left(2 \eta h+h^{2}\right) V_{n n}+O\left(h^{2}\right) .
$$

Dividing (28) by $\xi(h-\eta)+\eta$ and subtracting (29) divided by $h$, we obtain

$$
\frac{V_{c}-V_{a}}{\xi(h-\eta)+\eta}-\frac{V_{a}-V_{e}}{h}=\frac{1}{2}\left[\frac{h^{2}-2 h \eta}{\xi(h-\eta)+\eta}+h+2 \eta\right] V_{n n}+O\left(h^{2}\right) \text {. }
$$

Taking (24) minus twice (23) plus (26) and then dividing by $h^{2}$, we have

$$
\frac{V_{b}-2 V_{a}+V_{d}}{h^{2}}=V_{t t}+O(h) \text {. }
$$

Dividing (30) by the factor multiplying $V_{n n}$ in (30), then adding equation (31) and making use of $V_{n n}+V_{t t}=0$, we obtain

$$
\begin{array}{r}
V_{n n}+V_{t t}=2\left[\frac{h^{2}-2 h \eta}{\xi(n-\eta)+\eta}+h+2 \eta\right]^{-1}\left[\frac{V_{c}-V_{a}}{\xi(h-\eta)+\eta}-\frac{V_{a}-V_{c}}{h}\right] \\
+\frac{V_{b}-2 V_{a}+V_{d}}{h^{2}}+O(h)=0 .
\end{array}
$$

Equation (32) is our algebraic approximation in this case. It reduces to (22) when $\eta=0$. If we accept the idea that $V^{(i)}$ be continuable to neighboring meshpoints with terms through second order in a Taylor Series, as is implied by equations (23)-(27), then equation (32) is the unique algebraic representation of our problem for this case. Terms through second order are necessary in order that the local truncation error vanish as $h \rightarrow 0$. Some schemes which appear on the surface to represent our problem with local truncation error tending to zero when $h \rightarrow 0$ do not do so. Douglas, Garder, and Peaceman treated a moving boundary problem. According to Douglas, Garder, and Peaceman [10] to treat the case just considered we introduce an additional meshpoint at 0 . Then we can easily develop a divided difference approximation to Laplace's equation at $a$ in terms of $V_{a}, V_{b}$, $V_{0}, V_{d}, V_{e}$. We obtain

$$
\begin{aligned}
V_{n n}+V_{t t}=\frac{2}{h+\eta}\left[\frac{V_{0}-V_{a}}{\eta}-\frac{V_{a}-V_{e}}{h}\right] & \\
& +\frac{V_{b}-2 V_{a}+V_{d}}{h^{2}}+O(h)=0 .
\end{aligned}
$$

However, we now have an extra unknown $V_{0}$ so that we must have an additional equation. As our additional equation we choose an algebraic approximation to 
condition (d) of Problem A, namely

$$
D_{2} \frac{V_{c}-V_{0}}{h-\eta}=D_{1} \frac{V_{0}-V_{a}}{\eta}-\left[\frac{D_{1}}{2} \eta+\frac{D_{2}}{2}(h-\eta)\right] V_{n n}+O\left(h^{2}\right) .
$$

The truncation error term which is $O(h)$ is explicitly given in (34). We can compare (33), (34) with (32) by using (34) to eliminate $V_{0}$ from (33). Solving (34) for $\frac{V_{0}}{\eta}$ and then subtracting $\frac{V_{a}}{\eta}$ from both sides, we obtain

$$
\frac{V_{0}-V_{a}}{\eta}=\frac{V_{c}-V_{a}}{\xi(h-\eta)+\eta}+\frac{1}{2}\left[\frac{h-\eta+\xi \eta}{\xi(h-\eta)+\eta}\right](h-\eta) V_{n n}+O\left(h^{2}\right) .
$$

Substituting (35) into (33), we obtain

$$
\begin{aligned}
V_{n n}+V_{t t}=\frac{2}{h+\eta}\left[\frac{V_{c}-V_{a}}{\xi(h-\eta)+\eta}-\frac{V_{a}-V_{c}}{h}\right]+\frac{V_{b}-2 V_{a}+V_{d}}{h^{2}} \\
+\left[\frac{(h-\eta)+\xi \eta}{\eta+\xi(h-\eta)} \frac{h-\eta}{h+\eta}\right] V_{n n}+O(h)=0 .
\end{aligned}
$$

Equation (36) does not agree with (32) unless $\eta=\frac{h}{1-\xi}$ or $\eta=h$. Furthermore, in general, the local truncation error does not vanish as $h \rightarrow 0$. When $D_{1}=D_{2}$, it is easily shown that $V^{(1)}$ and $V^{(2)}$ are parts of the same harmonic function. (This is a theorem of potential theory, cf. Kellogg [11]). Since this is so, it would be expected that (32) and (36) should reduce to the ordinary five point formula for approximating the Laplace equation. (32) does this but (36) does not. The local truncation error in (36) remains finite when $h \rightarrow 0$ even for $\xi=1$. The fact that the local truncation error in (36) remains finite as $h \rightarrow 0$ does not mean that the error in the solution remains finite as $h \rightarrow 0$. The local truncation error may be regarded as a ficticious charge density distribution and the error in the solution as the potential of this distribution. As $h \rightarrow 0$ the local truncation error in (36) remains finite. Hence the error in the solution approaches the potential of a charged layer whose thickness approaches zero while the charge density in the layer remains finite. The potential of such a distribution will become everywhere arbitrarily small as the layer thickness goes to zero.

Equation (34) causes difficulties when the system of algebraic approximations is to be solved by an iteration method. The rate of convergence of most methods will tend to zero as $\eta \rightarrow 0$. Of course, the local truncation error in (36) may be reduced by approximating $V_{n n}$ by a finite difference approximation.

6. Algebraic approximations with more than five meshpoints. Except for special cases as we have discussed, no algebraic approximation with local truncation error which vanishes as $h \rightarrow 0$ is possible for the points adjacent to $\Gamma$ in terms of five meshpoints. If we make use of one additional neighbor and annex one additional equation to (10)-(14), then there is no difficulty in obtaining an algebraic approximation. We solve (10)-(13) together with our extra equation to obtain $V_{n n}$ and $V_{t t}$ in terms of values of $V$ at meshpoints. Then we substitute these expressions for $V_{n n}$ and $V_{t t}$ into equation (14). 
But which extra neighbor do we choose, and why? The problem becomes very unsymmetrical. If we make use of several additional neighbors, say the four corner points in figure 2 , then a symmetrical formulation is possible, but now the solution is no longer unique, there being more equations than necessary.

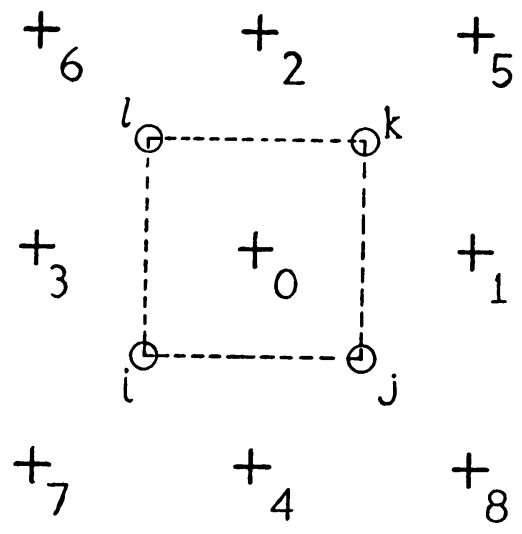

Figure 2.

In figure 2 we show the four neighbors of figure 1 relabeled " 1, , " 2, , " 3, ," " 4 ." We show the central meshpoint " $a$ " relabeled " 0 ," and we show the corner points " 5 ," "6," "7," "8." Let us suppose that the discontinuity intersects one of the line segments $0-1,0-2,0-3,0-4$, so that a special representation is necessary. We choose an arbitrary origin on the discontinuity and let the coordinates of the meshpoints relative to this origin be $\left(t_{i}, n_{i}\right), i=0,1, \cdots, 8$. Then the continuation equations analogous to (10)-(14) are

$$
\begin{aligned}
V_{i}-V_{0}=\left(\xi^{\delta} n_{i}-\xi^{\delta_{0}} n_{0}\right) V_{n}+\left(t_{i}-t_{0}\right) V_{t}+\frac{1}{2}\left(n_{i}{ }^{2}-n_{0}{ }^{2}\right) V_{n n} \\
+\left(\xi^{\delta_{i}} n_{i} t_{i}-\xi^{\delta_{0}} n_{0} t_{0}\right) V_{n t}+\frac{1}{2}\left(t_{i}{ }^{2}-t_{0}\right) V_{t t}, \quad i=1,2,3,4 .
\end{aligned}
$$

where $\delta_{i}=0$ when meshpoint $i$ is in $G_{1}, \delta_{i}=1$ when meshpoint $i$ is in $G_{2}$.

In addition we have the continuation equations for the corner points,

$$
\begin{aligned}
V_{i}-V_{0}=\left(\xi^{\delta_{i}} n_{i}-\xi^{\delta_{0}} n_{0}\right) V_{n}+\left(t_{i}-t_{0}\right) V_{t}+\frac{1}{2}\left(n_{i}{ }^{2}-n_{0}{ }^{2}\right) V_{n n} \\
+\left(\xi^{\delta_{i}} n_{i} t_{i}-\xi^{\delta} n_{0} t_{0}\right) V_{n t}+\frac{1}{2}\left(t_{i}{ }^{2}-t_{0}{ }^{2}\right) V_{t t}, \quad i=5,6,7,8 .
\end{aligned}
$$

The values of $V_{n n}$ and $V_{t t}$ obtained from any solution of (37), (38) are independent of where the origin is chosen on the discontinuity. To see this, suppose that the origin is displaced on the discontinuity so that $t_{i}$ is replaced by $t_{i}+\Delta t$. Then the coefficients of $V_{n}, V_{t}$ and $V_{n n}$ in (37), (38) are unchanged, but the coefficients of $V_{n t}, V_{t t}$ are increased by $\left(\xi^{\delta_{i}} n_{i}-\xi^{\delta_{0}} n_{0}\right) \Delta t$ and $\left(t_{i}-t_{0}\right) \Delta t$ respectively.

These latter quantities are proportional to the coefficients of $V_{n}$ and $V_{t}$. When we solve equations (37), (38) for $V_{n n}$ and $V_{t t}$, $\left(\xi^{\delta_{i}} n^{i}-\xi^{\delta_{0}} n_{0}\right) V_{n}$ and $\left(t_{i}-t_{0}\right) V_{t}$ must be eliminated, and when this is done the extra terms $\left(\xi^{\delta_{i}} n_{i}-\xi^{\delta_{0}} n_{0}\right) \Delta t V_{n t}$ and $\left(t_{i}-t_{0}\right) \Delta t V_{t t}$ will also drop out of the equations.

In order to obtain a non-singular set of 5 equations from (37), (38), we may choose the set of four equations in (37) and as a fifth equation choose

$$
V_{5}-V_{6}+V_{7}-V_{8}=a_{1} V_{n}+a_{2} V_{t}+a_{3} V_{n n}+a_{4} V_{n t}+a_{5} V_{t t},
$$


where $a_{1}, a_{2}, a_{3}, a_{4}, a_{5}$ are the coefficients of $V_{n}, V_{t}, V_{n n}, V_{n t}, V_{t t}$ obtained when we form equation (39) from (38), by taking the combination of equations (38) indicated by the left side of (39). The system of equations (37), (39) is a plausible set to use for obtaining an algebraic representation. Loosely speaking, (37) does not give a "well-set" determination of $V_{n t}$ while (39) does. $\left(V_{n t}\right.$ drops out of (37) when $\alpha=\frac{n \pi}{2}$.)

From the system (37), (39) we obtain (21), (22), and (32) in the respective limiting cases. Arms, Gates, and Zondek [12] have shown that extrapolated line relaxation may be used to solve difference equations involving nine-point formulas. Of course, it is not practical to solve (37), (39) to obtain a explicit formula analogous to (21), (22), (32) for the general case. However, it is not difficult to use a computer to calculate the coefficients in the algebraic representation for any given case. First we compute the matrix of coefficients of equations (37), (39) and then invert this matrix, and finally we combine elements of the inverse to obtain the values of the coefficients which multiply each $V_{i}$ in the algebraic representation of $V_{n n}+V_{t t}=0$.

Other methods for determining an algebraic approximation may be based on an integral formulation of our problem. Referring to figure 2, we let

$$
E_{l k}=\int_{l}^{k} D_{m} V_{y}^{(m)} d x, \quad \bar{E}_{j k}=\int_{j}^{k} D_{m} V_{x}^{(m)} d y,
$$

i.e., $E_{l k}$ is the line integral of $D_{m} V_{y}^{(m)}$ along the line $l k$ shown in figure $2, \bar{E}_{j k}$ the line integral from $j$ to $k$. Superscript $m$ will be one over a segment of the line of integration lying in region one, and will be two over a segment in region two. Then as may be shown from the conditions of Problem A, we have

$$
E_{l k}+\bar{E}_{j k}-\bar{E}_{i l}-E_{i j}=0 .
$$

The integrals $E_{l k}, \bar{E}_{j k}$, etc. may be approximated by algebraic approximations. Again values of $V$ at some of the corner points 5, 6, 7,8 will be required in making the approximations. Algebraic approximations based on (40), (41), have the advantage that the resulting equations obey a conservation law which is an algebraic analogue of Gauss's theorem $\oint_{m} \nabla V^{(m)} d \mathbf{S}=0$. In the case of moving boundary problems this method also has the advantage of giving a unique velocity for the moving boundary.

7. Curved Interfaces. If $\Gamma$ is an arc with continuous curvature, it may be approximated in neighborhoods by simple analytic arcs, for example, by osculating circles. The mesh spacing must be small enough so that the radial distances from the osculating circle to the nine meshpoints to be used in the algebraic approximation are less than and are preferably small compared to the radius of curvature. There are two ways in which we may obtain the continuation equations. One method consists in mapping the osculating circle onto a segment of the real axis in the $\gamma$-plane. This mapping is easily obtained. The mapping $Z=Z_{0}+R e^{\beta / R} e^{i(\alpha / R)}$ maps the real axis of the $\gamma=\alpha+i \beta$ plane for $0 \leq \alpha \leq 2 \pi$ into a circle with center at $Z_{0}$ and radius $R$ in the $Z$-plane. The inverse of this mapping will map a circle 
onto the segment $(0,2 \pi)$ of the real axis. Under the mapping the nine meshpoints to be used in the algebraic approximation are mapped into nine meshpoints in the $\gamma$ plane. The coordinates of these meshpoints in the $\gamma$ plane, relative to an origin on the real axis, are substituted into the continuation equations (37), (38).

A second method consists in expanding $V^{(i)}$ in a power series about a point $O$ on the osculating circle, and choosing $t$ to be arc length from $O$ on the osculating circle. We have as before $V_{n}^{(2)}=\xi V_{n}^{(1)}, V_{t}^{(2)}=V_{t}^{(1)}, V_{t t}^{(2)}=V_{t i}^{(1)}, V_{n t}^{(2)}=\xi V_{n t}^{(1)}$ but $V_{n n}^{(2)} \neq V_{n n}^{(1)}$. Expressing Laplace's equation in polar coordinates with origin at the center of curvature, we have

$$
V_{n n}^{(i)}+\frac{1}{r} V_{n}^{(i)}+V_{t i}^{(i)}=0
$$

whence it follows that

$$
V_{n n}^{(2)}=V_{n n}^{(1)}+\frac{1-\xi}{r} V_{n}^{(1)}
$$

Then the continuation equations (37), (38) become

$$
\begin{aligned}
V_{i}-V_{0}= & {\left[\left(\xi^{\delta_{i}} n_{i}+\delta_{i} \frac{1-\xi}{r} \frac{n_{i}{ }^{2}}{2}\right)-\left(\xi^{\delta_{0}} n_{0}+\delta_{0} \frac{1-\xi}{r} \frac{n_{0}{ }^{2}}{2}\right)\right] V_{n} } \\
& +\left(t_{i}-t_{0}\right) V_{t}+\frac{1}{2}\left(n_{i}{ }^{2}-n_{0}{ }^{2}\right) V_{n n}+\left(\xi^{\delta_{i}} n_{i} t_{i}-\xi^{\delta_{0}} n_{0} t_{0}\right) V_{n t} \\
& +\frac{1}{2}\left(t_{i}{ }^{2}-t_{0}{ }^{2}\right) V_{t t}, \quad i=1,2,3, \cdots, 8
\end{aligned}
$$

In (42), (43), (44) $r$ is positive if the center of curvature is in $G_{1}$, negative if in $G_{2}$. This follows because $n$ was the normal from $G_{1}$ into $G_{2}$.

8. Other equations. It can be shown that the power series method here described may be used to obtain algebraic approximations to analogues of Problem A for certain other elliptic or parabolic equations, for example, for the Helmholtz equation and for the diffusion equation.

Computer Usage Company, Inc.

18 East 41 st Street

New York, New York

1. J. H. Jeans, The Mathematical Theory of Electricity and Magnesism, Cambridge University Press, London, 1948.

2. W. R. SmYThE, Static and Dynamic Electricity, McGraw-Hill Book Co., New York, 1939.

3. R. E. KIDDER, "Motion of an interface between two immiscible liquids of unequal density in a porous solid," Jn. of Appl. Phys., v. 27, 1956, p. 1546-1548.

4. G. H. SHORTLEY \& R. WELLER, "The numerical solution of Laplace's equation," Jn. Appl. Phys., v. 9, 1938, p. 334-348.

5. R. V. VISWANATHAN, "Solution of Poisson's equation by relaxation method-normal gradient specified on curved boundaries," $M T A C$, v. XI, 1957, p. 67-78.

6. R. S. VARGA, "Numerical solution of the two-group diffusion equations in $x-y$ geometry," WAPD-159 AEC Research and Development Report, 1956, p. 8-12.

7. E. L. WACHSPRESS, "CURE: A generalized two-space-dimension multigroup coding for the IBM 704," KAPL-1724 AEC Research and Development Report, 1957, p. 20-28.

8. O. D. KellogG, Foundations of Potential Theory, Frederick Unger, New York, 1929, p. $286-315$.

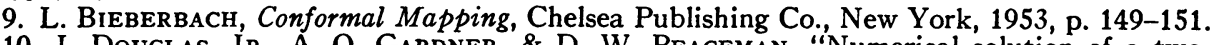

10. J. Douglas, JR., A. O. Gardner, \& D. W. Peaceman, "Numerical solution of a twodimensional moving boundary problem," Paper presented at Association for Computing Machinery Meeting, University of Houston, June 19-21, 1957. (Unpublished.)

11. O. D. KellogG, Op. Cit., p. 261.

12. L. J. ARMS, L. D. GATES, \& B. ZONDEK, "A method of block iteration," Soc. for Industrial and Applied Math., Jn., v. 4, 1956, p. 220-229. 